\title{
Glomerulonefritis antimembrana basal glomerular postrasplante renal en una adolescente con síndrome de Alport. Informe de caso
}

\section{Glomerular anti-basement membrane glomerulonephritis post-kidney transplant in a teenager with Alport syndrome. Case report}

Francisco José Arturo Calderón-Mendieta, ${ }^{1}$ Silvestre García-de la Puente, ${ }^{2}$ Nelly Gabriela Razo-Paredes, ${ }^{1}$ Dulce María Macías-Díaz, ${ }^{3}$ Michelle Alejandra Burciaga-Verduzco ${ }^{4}$

\begin{abstract}
Resumen
ANTECEDENTES: La glomerulonefritis antimembrana basal glomerular en el paciente trasplantado de riñón con síndrome de Alport es realmente excepcional. La mayoría de quienes lo padecen son hombres, en el primer año del postrasplante.

CASO CLínICO: Paciente femenina de 19 años, con diagnóstico histopatológico de síndrome de Alport a los 13 años, trasplantada a los 18 años. Tuvo un cuadro de disfunción súbita del injerto a los 20 meses después del trasplante. La disfunción renal fue resistente al tratamiento y tuvo pérdida del injerto a las 8 semanas. En la biopsia renal se diagnosticó: glomerulonefritis antimembrana basal glomerular.

CONCLUSIONES: La glomerulonefritis antimembrana basal glomerular es poco frecuente en el paciente trasplantado con síndrome de Alport; aun así, debe considerarse una opción diagnóstica ante la falla del tratamiento habitual. La generación de nuevas medidas terapéuticas efectivas, por el riesgo elevado de pérdida del injerto, es por demás importante.
\end{abstract}

PALABRAS CLAVES: Síndrome de Alport; glomerulonefritis; trasplante renal; glomérulo renal.

\section{Abstract}

BACKGROUND: The overall incidence of post-transplant antiglomerular basement membrane in patient transplanted from Alport syndrome is about $5 \%$, being more frequent in men and in the first year of the transplant.

CLINICAL CASE: Female patient of 19 years, with histopathological diagnosis of Alport syndrome at 13 years, transplanted at 18 years. He had a picture of sudden graft dysfunction at 20 months after the transplant. Renal dysfunction was resistant to treatment and had graft loss at 8 weeks. The renal biopsy was diagnosed: glomerular basal glomerulonephritis.

CONCLUSIONS: Although antiglomerular basement membrane glomerulonephritis it's a low frequency entity in the setting of patient transplanted from Alport Syndrome, it has to raise the possibility of this disease when the usual therapeutic fail, because there is a high rate of graft loss $(75 \%)$ the development of new strategies for this type of patients is needed.

KEYWORDS: Alport syndrome; Glomerulonephritis; Kidney Transplantation; Kidney Glomerulus.

\footnotetext{
Nefrólogo pediatra, Hospital ISSSTECALI, Mexicali, Baja California, México. 2 Jefe del Departamento de Metodología de la Investigación, Instituto $\mathrm{Na}$ cional de Pediatría, Ciudad de México. ${ }^{3}$ Nefropatóloga, Departamento de Patología, Hospital Centenario Migue Hidalgo, Aguascalientes, Aguascalientes, México.

${ }^{4}$ Médico interno de pregrado, Hospital ISSSTECALI, Mexicali, Baja California, México
}

Recibido: 19 de septiembre 2019

Aceptado: 18 de febrero 2020

Correspondencia

Francisco José Arturo

Calderón Mendieta

fjacm76@gmail.com

Este artículo debe citarse como Calderón Mendieta FJA, García de la Puente S, Razo Paredes NG, Macías Díaz DM, Burciaga Verduzco MA, Glomerulonefritis antimembrana basal glomerular postrasplante renal en una adolescente con síndrome de Alport. Informe de caso. Acta Pediatr Méx 2020;41(2):66-71. 


\section{INTRODUCCIÓN}

La glomerulonefritis antimembrana basal glomerular es un tipo de glomerulonefritis rápidamente progresiva, caracterizada clínicamente por disfunción súbita de la función renal, con hematuria y proteinuria subnefrótica. Coexisten anticuerpos IgG antimembrana basal glomerular dirigidos contra el dominio no colágeno de las cadenas alfa $(\alpha 3, \alpha 4, \alpha 5)$ de la colágena tipo IV. ${ }^{1}$

El síndrome de Alport es una alteración genética caracterizada por defectos en la producción de las cadenas alfa $(\alpha 3, \alpha 4, \alpha 5)$ de la colágena tipo IV que implica daño ocular, pérdida de la audición neurosensorial e insuficiencia renal progresiva. ${ }^{2}$ En la mayoría de los casos el trasplante renal es una de las opciones de tratamiento porque debido a la ausencia de exposición a los antígenos de cadenas alfa de la colágena tipo IV en los riñones nativos, el injerto renal es capaz de provocar una respuesta inmunitaria, pérdida de la tolerancia y formación de anticuerpos antimembrana basal glomerular. La incidencia de glomerulonefritis antimembrana basal glomerular en el paciente postrasplantado, con síndrome de Alport, es de 2 a $5 \%{ }^{3}$
Enseguida se expone el caso de una adolescente con síndrome de Alport que resultó con glomerulonefritis antimembrana basal glomerular luego del traspante.

\section{CASO CLÍNICO}

Paciente femenina de 19 años, con antecedentes de hipoacusia neurosensorial a los 3 años y astigmatismo a $\operatorname{los} 7, \sin$ consanguinidad en los padres. La enviaron al servicio de Nefrología debido a la proteinuria en límite nefrótico y hematuria, síndrome de Alport a los 13 años (2012) por biopsia renal percutánea que reportó: microscopia óptica con ligero engrosamiento irregular de la membrana basal glomerular sin esclerosis, fibrosis intersticial, atrofia tubular leve y arteriolonefroesclerosis. Inmunofluorescencia sin depósito de inmunocomplejos. Microscopia electrónica: ovillo glomerular con irregularidad en el grosor de la membrana basal, con segmentos delgados que alternan con otros engrosados. En los segmentos mejor conservados, la membrana pierde su propiedad trilaminar $y$, en su lugar, el tejido es de aspecto en red. De manera focal también hubo simplificación de procesos podocíticos y expansión mesangial. Figura 1

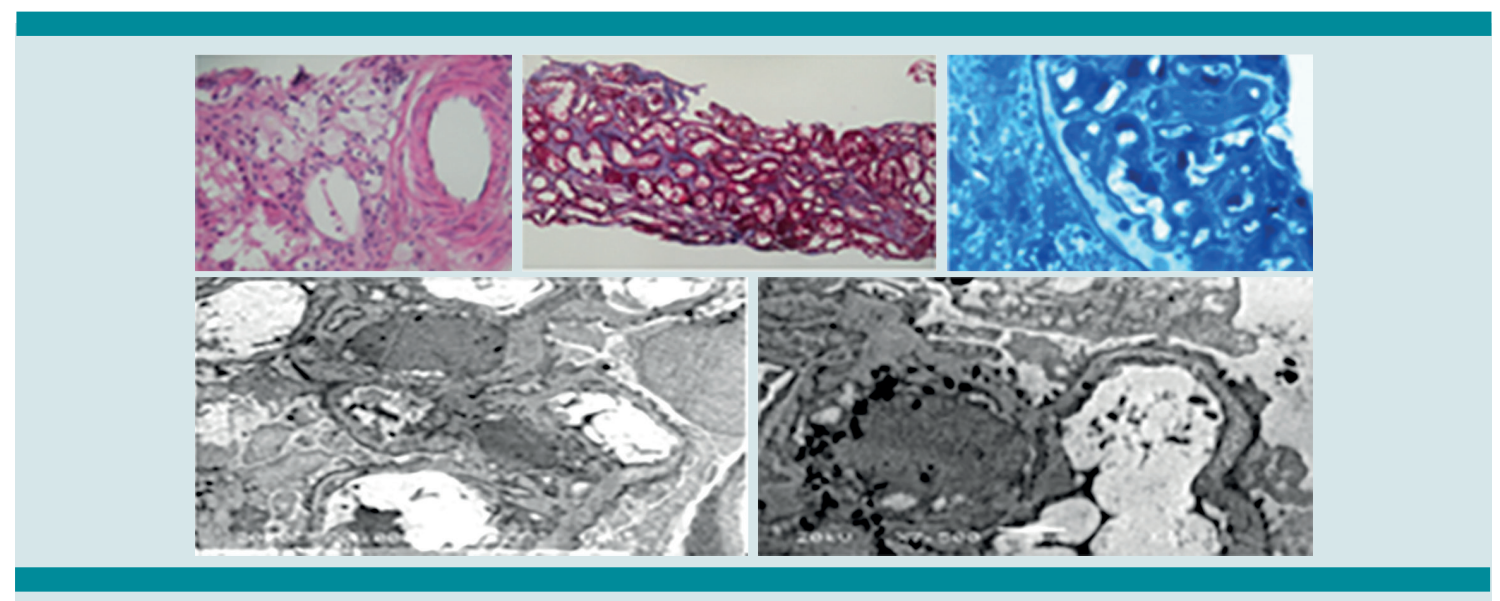

Figura 1. Microscopia electrónica en la que se observan segmentos de la membrana basal glomerular con disminución de su grosor, alternando con segmentos engrosados, pérdida de la propiedad trilaminar y fusión de los pedicelos de los podocitos. 
El deterioro de la función renal continuó. En enero de 2017 la creatinina se reportó en valores de $4.8 \mathrm{mg} / \mathrm{dL}$. Se efectuó el trasplante renal, de donador vivo relacionado de 46 años (padre); compartían cuatro antígenos de clase II del complejo mayor de histocompatibilidad (Cuadro 1). Se encontraron: injerto renal izquierdo, con una vena y una arteria renal. Tiempo de isquemia caliente de 5 minutos. Tiempo de isquemia fría 50 minutos, diuresis espontánea a los 4 minutos. Durante el transoperatorio se le transfundió un paquete globular, con filtro leucorreductor. Inducción con basiliximab y tres pulsos de metilprednisolona en días consecutivos. Recibió tratamiento postrasplante con $4 \mathrm{mg}$ al día de tacrolimus, $1 \mathrm{~g}$ al día de micofenolato de mofetil y $5 \mathrm{mg}$ al día de prednisona. Tres meses después, la tasa de filtrado glomerular se estimó en $92 \mathrm{~mL} / \mathrm{min}$.

Cuadro 1. Prueba de histocompatibilidad

\begin{tabular}{|l|ll|ll|}
\hline Paciente & \multicolumn{2}{|c|}{ Clase I } & \multicolumn{2}{c|}{ Clase II } \\
\hline Receptor & A01 & B08 & DR07 & DQ09 \\
& A25 & B58 & DR08 & DQ04 \\
Donador & A25 & B58 & DR08 & DQ04 \\
& A02 & B51 & DR07 & DQ09 \\
\hline
\end{tabular}

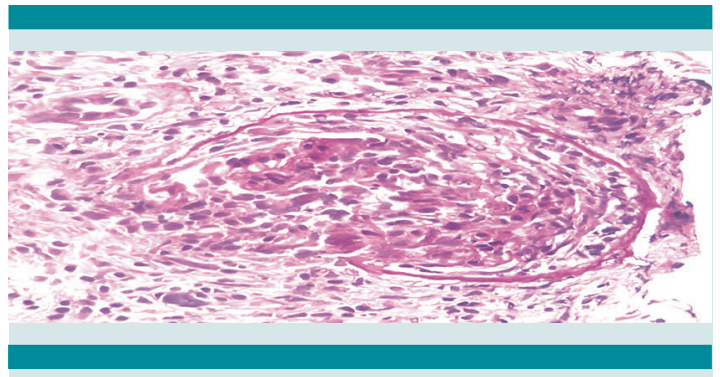

Figura 2. Fotomicrografía 40x tinción de PAS. Se observa un glomérulo con pérdida parcial de la cápsula de Bowman e infiltrado por linfocitos, células plasmáticas y algunos neutrófilos. El espacio urinario está ocupado por la proliferación de células epiteliales parietales, y algunos linfocitos y células plasmáticas. El ovillo glomerular está retraído, con membrana basal corrugada.
A los 20 meses del trasplante (agosto de 2018) se hospitalizó debido a síntomas compatibles con rechazo agudo del injerto renal, incremento súbito de la creatinina y disminución del volumen urinario. Se trató con pulsos de metilprednisolona, con pobre respuesta. Se le aplicó ciclofosfamida, tres recambios plasmáticos y biopsia renal (Figuras 2 y 3) y se reportó necrosis segmentaria y proliferación extracapilar difusa, nefritis túbulointersticial grave. En la inmunofluorescencia se encontraron depósitos lineales de lgG y C3 en la membrana basal glomerular y tubular compatible

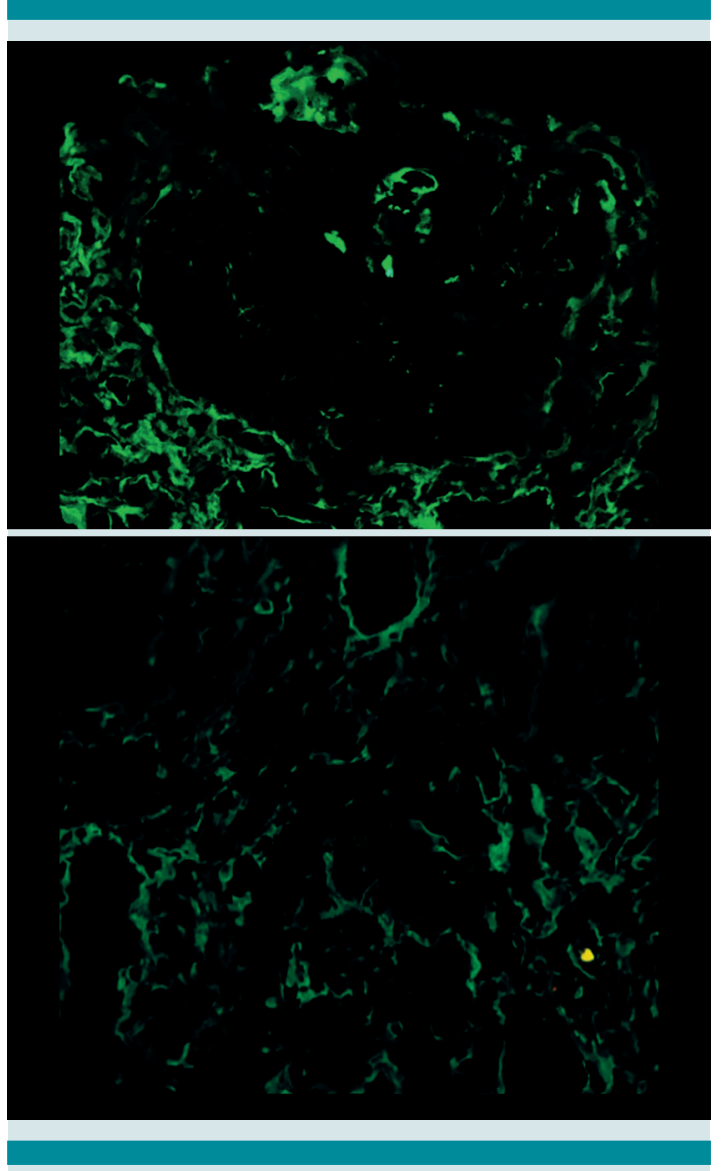

Figura 3. Fotomicrografía 40x. Con lámpara de inmunofluorescencia, IgG positiva lineal en una asa capilar conservada, fragmentos de membrana basal, cápsula de Bowman y membrana basal tubular. 
con glomerulonefritis por anticuerpos antimembrana basal glomerular postrasplante.

Se inició la hemodiálisis y la evolución fue tórpida y la condujo a la pérdida del injerto a las 8 semanas posteriores. Las concentraciones séricas de anticuerpos antimembrana basal glomerular, por técnica de ELISA, se reportaron mayores de $200 \mathrm{UR} / \mathrm{mL}$. Inicialmente en hemodiálisis y posteriormente se envió a diálisis peritoneal continua ambulatoria, donde permanece actualmente con un volumen urinario residual de $500 \mathrm{~mL}$.

\section{DISCUSIÓN}

El síndrome de Alport es una alteración genética caracterizada por deficiencia en la producción de las cadenas de colágena, éstas forman un heterotrímero principalmente en la red de la membrana basal glomerular, coclear, córnea, cápsula de lente y retina. Su patrón de herencia se encuentra, según distintas series, distribuido en: ligado al cromosoma X incluso en 85\%,10\% para el patrón autosómico recesivo y $5 \%$ para el autosómico dominante. ${ }^{2}$

La heterogeneidad de la manifestación clínica depende de la forma de trasmisión genética. Los varones con síndrome de Alport ligado al cromosoma X casi siempre resultan con enfermedad renal crónica severa en la segunda década de la vida, pérdida de la audición y alteraciones oftalmológicas. En cambio, las mujeres tienen una evolución más lenta y algunas no llegan a la insuficiencia renal. Las que tienen patrón autosómico recesivo cursan con hematuria y proteinuria de forma común en la adolescencia, con evolución hacia enfermedad renal crónica alrededor de la segunda década de la vida. Las alteraciones oftalmológicas más frecuentes son: lenticono y retinopatía periférica. ${ }^{2}$ Independientemente del género, la mayoría de los pacientes con patrón autosómico recesivo resultan con enfermedad renal crónica y pérdida de la audición en etapas tempranas de la vida. ${ }^{2}$

La descripción inicial de la enfermedad la publicó Arthur Cecil Alport, en 1927, en una familia británica, con tres generaciones afectadas por problemas renales. En México existe poca información respecto de la incidencia, prevalencia y patrones de herencia del síndrome de Alport. En 1981 García Delgadillo y Gordillo Paniagua describieron el caso de cuatro familias no relacionadas (Tabasco, Puebla, Michoacán y Durango) con patrón hereditario autosómico dominante. ${ }^{4}$ Cruz Robles y colaboradores reportaron, en 1999, tres nuevas mutaciones en el gen COL4A5 en pacientes mexicanos. ${ }^{5}$

En este caso no existe antecedente familiar de la enfermedad en generaciones previas, ni consanguineidad por parte de los padres, por lo que la manifestación clínica se caracterizó por evolución rápida de los síntomas, hipoacusia a los tres años, proteinuria en rango nefrótico a los 13 años, enfermedad renal crónica avanzada y trasplante a los 15 años. Es altamente compatible con el patrón de AR. Este tipo de herencia se reporta en 1 por cada 40,000 individuos; la relación hombre-mujer es de 1:1. Los genes afectados son la mutación en ambos alelos (homocigotos) del COL4A3 o del COL4A4 en el cromosoma 2 q $35-37 .^{2}$

En las guías de tratamiento de los pacientes con síndrome de Alport y enfermedad de membranas delgadas del 2013 se recomienda el diagnóstico genético; sin embargo, en nuestro caso no fue posible practicarlo. ${ }^{6}$

El pronóstico del paciente con síndrome de Alport trasplantado es igual o mejor que para otras enfermedades hereditarias. ${ }^{6}$ En 1995 los estudios de colaboración de trasplante renal pediátrico de Estados Unidos refieren la supervivencia del injerto a 2 años de 95\% de donador vivo y de 
$70 \%$ de donador cadavérico versus 89 y $74 \%$ respectivamente de pacientes trasplantados con malformaciones urológicas. ${ }^{7}$ En 2012, Temme y colaboradores, de la Asociación Renal Europea (ERA) y la Asociación Europea de Diálisis y Trasplante (EDRTA) reportaron que los varones adultos trasplantados por síndrome de Alport tenían mayor supervivencia, y también el injerto, corregida para edad, fecha de trasplante y donador. ${ }^{8}$ Una publicación más reciente en Irlanda identificó a 51 pacientes con síndrome de Alport a lo largo de 33 años con supervivencia del injerto de 20 años en $70 \%$ de los pacientes con síndrome de Alport vs $44.8 \%$ para otras enfermedades renales; en esa serie no se identificaron casos de glomerulonefritis antimembrana basal glomerular postrasplante. ${ }^{9}$ Gumber y su grupo (2012) reportaron una serie de 31 pacientes, $21 \%$ con disfunción aguda del injerto en la que se identificaron 4 pacientes (12\%) con glomerulonefritis antimembrana basal glomerular. ${ }^{10}$ Gillion, en Bélgica, publicó (2018) el reporte de 43 años en el que identificaron 73 pacientes trasplantados por síndrome de Alport, solo 1.4\% de ellos resultó con glomerulonefritis antimembrana basal glomerular. ${ }^{11}$ Katsuma y su equipo (2018) reportaron supervivencia del injerto de pacientes trasplantados con síndrome de Alport de $100 \%$ en el primer año, $86.7 \%$ a los 5 años y $69 \%$ a los 10 años, comparado con otras causas de enfermedad renal crónica que reportaron 95,77 y $64 \%$ al primer, quinto y décimo año postrasplante, respectivamente. La diferencia no fue significativa y las causas de pérdida del injerto fueron originadas, principalmente, por rechazo crónico $(66.7 \%)$ y pobre apego al tratamiento (16\%), sin identificar glomerulonefritis antimembrana basal glomerular en el grupo con síndrome de Alport. ${ }^{12}$

En la mayor parte de las revisiones, la glomerulonefritis antimembrana basal glomerular se asocia con pérdida del injerto en el síndrome de Alport (75\%). Se informa que su incidencia alcanza 2 a $5 \%$ de los pacientes trasplantados, sobre todo relacionada con pacientes con síndrome de Alport ligado al cromosoma X. 3,11,13

Los casos de enfermedad antimembrana basal glomerular relacionada con mujeres con patrón autosómico recesivo son excepcionalmente ra$\operatorname{ros}^{2,6}$ debido a que la fisiopatología habitual de la enfermedad antimembrana basal glomerular corresponde a la ausencia del antígeno COL4A5, que solo se relaciona con el SALX. En el caso del patrón autosómico recesivo, el antígeno COL4A3 en el injerto del donante es el responsable de la generación de anticuerpos de novo postrasplante, que es lo que genera la glomerulonefritis antimembrana basal glomerular. Este antígeno también es el epítopo responsable de la enfermedad de Goodpasture y la antimembrana basal glomerular primaria. ${ }^{14}$ La mayoría de los pacientes padecen la enfermedad en el primer año luego del trasplante, aunque puede aparecer en cualquier momento de la vida del injerto, como sucedió en la paciente motivo de esta comunicación que inició al año 7 meses del trasplante. La manifestación clínica puede ser la elevación súbita de la creatinina como única alteración o glomerulonefritis rápidamente progresiva. ${ }^{1}$ Es una enfermedad que con frecuencia se relaciona con pérdida del injerto, incluso en $75 \%$ de los pacientes, a pesar de múltiples medidas de tratamiento médico. Hay mayor posibilidad de padecer la enfermedad en pacientes con una gran deleción génica versus los que solo tienen alteraciones muy específicas, infiriendo que el sistema inmunitario nunca ha estado expuesto a la proteína madura responsable ${ }^{1,3}$

El diagnóstico se sospecha en la manifestación clínica, los hallazgos histopatológicos y en la detección de concentraciones elevadas de anticuerpos antimembrana basal glomerular en la mayoría de los casos. En la paciente del caso se detectaron más de $200 \mathrm{UR} / \mathrm{mL}$ de anticuerpos antimembrana basal glomerular con técnica de 
ELISA, que es menos específica que Western Blot. ${ }^{15}$ La biopsia del injerto suele revelar hipercelularidad extracapilar, con necrosis fibrinoide segmentaria, ruptura de la membrana basal glomerular. La inmunofluorescencia demuestra depósito linear de IgG y C3 en la membrana basal glomerular. ${ }^{1}$

La terapia con pulsos de metilprednisolona, ciclofosfamida y recambio plasmático suele ser la primera línea de tratamiento, aunque los desenlaces no son favorables, muy similar a lo que se le indicó a la paciente, sin conseguir mejoría de la función del injerto.

La mayoría de los pacientes con glomerulonefritis antimembrana basal glomerular de novo postrasplante continúan con recurrencia en los siguientes trasplantes, incluso con concentraciones séricas bajas o indetectables de anticuerpos antimembrana basal glomerular en suero. Lo común es que la enfermedad evolucione en meses o años en el primer trasplante, en semanas en el segundo trasplante y en días en el tercero. ${ }^{3}$

\section{CONCLUSIONES}

La glomerulonefritis antimembrana basal glomerular es poco frecuente pero debe sospecharse en pacientes trasplantados con disfunción súbita del injerto, con antecedente de síndrome de Alport. Puesto que la pérdida del injerto es muy frecuente y la recurrencia en los siguientes trasplantes es común, es primordial el inicio temprano de tratamiento, y la búsqueda de nuevas medidas terapéuticas antirrechazo efectivas.

\section{REFERENCIAS}

1. McAdoo SP, Pusey CD. Anti-Glomerular Basement Membrane Disease. Clin J Am Soc Nephrol 2017; 12 (7): 1162-72. https://doi.org/10.2215/CJN.01380217
2. Savige J, et al. Alport syndrome in womens and girls. Clin J Am Soc Nephrol 2016; 11 (9): 1713-20. https://doi. org/10.2215/CJN.00580116

3. Browne $G$, Bret al. Retransplantation in Alport post-transplant anti-GBM disease. Kidney Int 2004; 65 (2): 675-81. doi: 10.1111/j.1523-1755.2004.00428.x

4. García-Delgado C, Gordillo-Paniagua G. Epidemiological study in 4 families units with Alport Syndrome. Bol Med Hosp Infant Mex 1981; 38 (6): 887-902.

5. Cruz Robles D, et al. Three Novel Mutations in the COL4A5 gene in Mexican Alport Syndrome Patients. Clin Genet 1999; 56:242-3.

6. Savige J, et al. Expert guidelines for the management of Alport syndrome and thin basement membrane nephropathy. J Am Soc Nephrol 2013; 24 (3): 364-75. doi: 10.1681/ ASN.2012020148.

7. Kasthan $\mathrm{CE}$, et al. Renal allograft survival according to primary diagnosis. A report of the North American Pediatric Renal Transplant Cooperative Study. Pediatr Nephrol 1995; 9: 679-84.

8. Temme J, et al. Outcomes of male patients with Alport syndrome undergoing renal replacement therapy. Clin J Am Soc Nephrol 2012; 7 (12): 1969-76. doi: 10.2215/ CJN.02190312

9. Kelly $\mathrm{YP}$, et al. Outcomes of kidney transplantation in Alport syndrome compared with other forms of renal disease. Ren Fail 2017; 39 (1): 290-3. doi: 10.1080/0886022X.2016.1262266.

10. Gumber MR, et al. Outcome of renal transplantation in Alport syndrome: a single-center experience. Transplant Proc 2012; 44 (1): 261-3. doi: 10.1016/j.transproceed.2011.11.035.

11. Gillion V, et al. Genotype and outcome after kidney transplantation in Alport Syndrome. Kidney Int Rep 2018; 3 (3): 652-60. doi: 10.1016/j.ekir.2018.01.008.

12. Katsuma $A$, et al. Long-term survival in Japanese renal transplant recipients with Alport syndrome: a retrospective study. BMC Nephrol 2018; 19 (1): 249. doi: 10.1186/ s12882-018-1052-9

13. Clifford K. Renal transplantation in patients with Alport syndrome: patient selection, outcomes, and donor evaluation. Int J Nephrol Renovasc Dis 2018; 11: 267-70. doi: 10.2147/ IJNRD.S150539

14. Rheault M. Women and Alport Syndrome. Pediatr Nephrol 2012; 27(1): 41-6. doi: 10.1007/s00467-011-1836-7

15. Clifford, K. Renal transplantation in patients with Alport syndrome. Pediatr Transplant 2006; 10 (6): 6. 